\title{
Uncertainty and International Climate Change Negotiations
}

\author{
Yiyong Cai · Warwick McKibbin
}

Received: 6 July 2014 / Accepted: 20 November 2014 / Published online: 14 February 2015

(C) Società Italiana degli Economisti (Italian Economic Association) 2015

\begin{abstract}
Because issues associated with climate change are historically unprecedented and thus policymakers do not have a prior distribution over possible outcomes, the usual theoretical approach based on governments maximizing expected utility may not be suitable for analysing climate policy choice. Under an alternative plausible assumption that policymakers act strategically but choose the policy that incurs the highest possible gain in the worst-case scenario, this paper shows how collectivism can be inferior to unilateralism in both carbon mitigation and economic loss minimization. Our proposed approach provides a possible explanation for several paradoxes in the existing literature in relation to uncertainty and climate policy negotiation. It also provides an analytical framework that can be applied to numerical simulations of international climate policy games.
\end{abstract}

Keywords Climate change - Policy game - International negotiation - Robust optimal control $\cdot$ Maxmin

JEL Classification $\quad \mathrm{C} 70 \cdot \mathrm{F} 51 \cdot \mathrm{Q} 54$

This study was undertaken while Yiyong Cai worked with the Australian National University.

Y. Cai $(\otimes)$

CSIRO Oceans and Atmosphere Flagship, Commonwealth Scientific and Industrial Research Organisation (CSIRO), Forestry House, Building 2, Wilf Crane Crescent, Yarralumla, ACT 2601, Australia

e-mail: yiyong.cai@csiro.au

Y. Cai · W. McKibbin

Centre for Applied Macroeconomic Analysis, Crawford School of Public Policy, Australian National

University, J.G. Crawford Building, Lennox Crossing, Acton, ACT 0200, Australia

W. McKibbin

The Brookings Institution, 1775 Massachusetts Ave NW,

Washington, DC 20036, USA 


\section{Introduction}

The rapidly increasing concentration of greenhouse gases in the atmosphere due to human activity is believed by many to be a key contributing factor in climate change (Field et al. 2011). The emission of greenhouse gases is generally considered as a market failure which requires global collective actions to adress (Hoel 1991; Uzawa 2003). Governments are well aware of the argument that if emissions of greenhouse gases continue to follow recent trends, the world may be at risk of catastrophic disasters in the decades to come. Nevertheless, global efforts towards greenhouse gas mitigation keep running into delay as is seen at the numerous meetings of the $\mathrm{UNFCCC}^{1}$ Conferences of Parties.

Although the public good aspect of climate change is at the forefront of most economic analyses of climate change, it is uncertainty that is another important characteristic of the policy problem. Many of the issues surrounding climate change are historically unprecedented. This makes formulating national policy extremely difficult. It also creates problems for analysing international policy in the usual context of a game being played between countries. The standard approach to policy choice is based on maximizing expected utility, but it becomes problematic for analysing climate policy choice when policymakers do not have a well defined prior distribution over possible outcomes (Kunreuther et al. 2013). In particular, the exected utility approach has difficulty explaining the failure of international climate policy coordination. While Ulph and Ulph (1997) shows that uncertainty facilitates coordination based on the theory of expected utility, this has been challenged by the laboratory findings of Barrett and Dannenberg $(2012,2014)$ that collective action fails when there is large uncertainty regarding the threshold for "dangerous" climate change.

Various alternatives to expected utility maximization have been suggested for analyzing climate policies under uncertainty. For example, the "Limited Degree of Confidence" criterion maximizes a weighted average of the expected utility in all possible cases and the expected utility in the worst $1 \%$ scenarios. The "Safety First" criterion maximizes the expected utility with the constraint that the probability of reaching some lower-bound utility is bounded (see Hall et al. 2012; McInerney et al. 2012). However, these approaches still require a prior distribution over possible outcomes, and both predict even more mitigation than maximizing expected utility.

Other scholars have argued in favor of the non-probabilistic "maxmin" decision rule for maximizing the economic welfare for a worst case climate scenario (see e.g., Froyn 2005; Funke and Paetz 2011). Climate change is due to the cumulative nature of carbon concentration (Ulph and Ulph 1997; Weitzman 2012). Therefore, if the consequences predicted by many scientists are to be avoided, precautious decisions need to be made now to reduce greenhouse gas emissions before the probabilities are well known. This is exemplified in the 2010 BBC Radio 4 interview of former UK Prime Minsister Tony Blair ${ }^{2}$ : It doesn't need to be certain for us to act ... if you find out 2030 or 2040

\footnotetext{
1 UNFCCC stands for the United Nations Framework Convention on Climate Change.

2 Tony Blair was the British Prime Minister from 1997 to 2007. He used the United Kingdom's G8 presidency in 2005 to address the issue of climate change.
} 
'that was a real problem, we should have dealt with that', you're going to pay a pretty heavy price in history. Under this degree of uncertainty, policymakers are faced with making decisions while knowing that the consequences of their policy choices under the worst case scenario if realized may be dire. The "maxmin" perspective of climate uncertainty justifies strong mitigation action (Funke and Paetz 2011), and again, fails to account for the stalemate of international climate policy.

The problem facing policymakers is actually even more complex than the quote of Tony Blair suggests. Apart from climate uncertainty, policymakers are also faced with economic uncertainty surrounding the costs of carbon mitigation. This form of uncertainty is reflected by former Australian Prime Minister John Howard's ${ }^{3} 2006$ speech to the Business Council of Australia where he stated: (Ratifying Kyoto) could have damaged the comparative advantage this country enjoyed ... I do not intend to preside over policy changes in this area that are going to rob Australia of her competitive advantage .... From a practical point of view, the "maxmin" decision rule is not useful if it does not account for the costs of carbon mitigation, which play an important role in breaking the deadlock in international negotiation. An over-cautious mitigation policy could lead to a drain of public finance.

In this paper we expand the "maxmin" approach to address both climate and economic uncertainties in the making of international climate policies. We follow van den Broek et al. (2003), Jiménez-Lizárraga and Poznyak (2007, 2012) to apply the theory of robust optimal control in a multi-player game context. We assume that policymakers' preferences over economic outputs under climate change can be represented by some utility functions. Collectivism refers to the existence of a political regime under which policymakers set their policy instruments to maximize their joint utility, the "global welfare". In contrast, unilateralism is the status quo in which policymakers are only concerned about their individual utility. In the process of negotiation, policymakers firstly choose whether to agree on collective action, and consequently formulate policies that secure the highest payoff in the least-favorable situation. This means that there will not be any regrets of policymakers under the worst-case scenario.

The idea is modelled by introducing an additional player called "nature"4 into the game between countries. Nature strategically chooses the least-favorable combination of climatic and economic events (see e.g., Zhou et al. 1996; Basar and Bernhard 2008; Basar and Olsder 1999; Hansen and Sargent 2008; Funke and Paetz 2011). If the policymakers increase efforts to mitigate, nature will decrease the impacts of climate change but increase the costs of mitigation, leading to the policymakers' regret of over-mitigation. In contrast, if the policymakers decrease efforts to mitigate, nature will increase the impacts of climate change but decrease the costs of mitigation, leading to the policymakers' regret of under-mitigation. This additional player confounds the policy response of countries in the climate policy game and thus captures policymakers' concerns about worst-case climate and economic outcomes.

\footnotetext{
3 John Howard was the Australian Prime Minister from 1996 to 2007 . He refused to ratify the Kyoto Protocol.

4 Sometimes called "evil nature" in the literature.
} 
With a very simple model that features the interaction between economic output, greenhouse emissions and climate policies, this paper shows that, in the context of the assumed goals of policymakers, collectivism is not generally optimal. Indeed, there are cases when unilateralism is superior for both carbon mitigation and economic loss minimization. Our proposed approach provides a plausible framework for explaining several mutual contrasting theories and empirical findings in the recent literature on uncertainty and climate policy negotiation (e.g., Barrett and Dannenberg 2012, 2014; Barrett 2013; Vasconcelos et al. 2013). It also provides an analytical framework that can be applied to numerical simulations of international climate policy games.

\section{The Model}

\subsection{Overview}

We consider a model with two countries, home $h$ and foreign $f$. Each country produces one differentiated good, the process of which generates emissions. Both home and foreign products are exported to the world market, and supply is demand-driven. Emissions are public bads (negative public goods). A carbon tax is the only policy instrument of policymakers, although the framework can also be extended to any other carbon policy that has equivalent effects; for example, the emission permit price in a cap-and-trade system. Idiosyncratic uncertainties exist in relation to the market and catastrophic climate change.

To avoid the effect of size and its effect on relative gains, the home and foreign economies are constructed to be symmetric, both having identical technology and preferences. Subjective beliefs about uncertainties are also assumed to be symmetric. In most of what follows, only the structural equations of the home country will be specified, with the understanding that comparable equations hold in the foreign country. Lower case variables represent the logrithmics of their upper-case equivalent variable names, unless otherwise specified.

This paper uses a stylized model with two symmetric countries only in order to address the effects of uncertainty in extreme scenarios on decision making. Such a theoretical abstraction enables us to focus on the policy problem of particular interest. We recognize that countries are asymmetric in the real world. ${ }^{5}$ They differ in market structure, climate vulnerability, carbon footprints and economic status. These are all important factors contributing to the deadlock of global carbon mitigation. Furthermore, our model can be extended to a finite-time dynamic game without much difficulty. For integrated assessment of international climate policy, it is therefore desirable to incorporate the methodology that is proposed in this paper into multicountry dynamic economic models such as RICE (Nordhaus and Yang 1996), CWS (Eyckmans and Bertrand 2000), WITCH (Bosetti et al. 2007, 2008), or G-Cubed (McKibbin and Wilcoxen 2013).

\footnotetext{
5 Assuming asymmetric home and foreign economies will not fundamentally alter the analytical results of this paper. Such complication will also make the model less tractable.
} 


\subsection{Output and Emissions}

We use a simple goods market that is similar to Dornbusch (1976). Both home and foreign goods are produced at cost 1 , and the gross tax rates (i.e., carbon taxes) $R_{h}, R_{f}$ coincide with the prices $P_{h}, P_{f}$. Outputs $Q_{h}, Q_{f}$ are driven by demand. Letting lower case variables represent the logrithmics of their upper-case counters, we assume the demand for home goods is

$$
q_{h}=r_{f}-r_{h}-w_{h} r_{h}
$$

Here, $r_{f}-r_{h}$ is the trade effect that is negatively related to the relative price of home and foreign goods; and $w_{h} r_{h}$ is the unmodeled effects of tax (see Brainard 1967) which captures changes of consumer preference, income and rate of returns on capital (see Dornbusch 1976, p. 1164, Eq. 7). The realization of $w_{h}$ can be arbitrarily small or large, and it is unknown to the home policymaker. Symmetrically, the foreign output is

$$
q_{f}=r_{h}-r_{f}-w_{f} r_{f} \text {. }
$$

Adding Eqs. (1) and (2), we have

$$
q_{h}+q_{f}=-w_{h} r_{h}-w_{f} r_{f}
$$

We note that the home output loss as a consequence of carbon pricing does not necessarily accrue to the foreign output gain unless $w_{h}=w_{f}=0$, and vice versa. In other words, there could be deadweight loss of global output after cancelling the trade effects of carbon pricing. Intuitively, imposing a price on carbon will lead to a restructuring of economic activities, such as investment in new infrastructure, altered patterns of energy consumption, redeployment and reskilling of labour, and the dissemination of new technologies. This increases the overall cost of industrial production and household consumption in the short term, and will usually lead to a decline of output and national income.

With regard to home emissions of greenhouse gases, $E_{h}$, we assume the following

$$
e_{h}=r_{f}-(1+\tau) r_{h}
$$

Here the parameter $\tau$ is strictly positive. In the case of symmetric action $r_{h}=r_{f}$ which rules out the trade effects of carbon pricing, we have

$$
\tau=-\frac{\partial e_{h}}{\partial r_{h}}=-\frac{\partial \ln E_{h}}{\partial \ln R_{h}}=-\frac{\partial \ln E_{h}}{\partial \ln P_{h}} .
$$

Therefore, $\tau$ could be understood as the price elasticity of energy demand if we assume a linear relation between energy consumption and emissions.

Note that Eq. (3) is a simplistic representation of the complex relationship between carbon pricing and emissions. This is a good starting point. In future research we do, however, acknowledge the importance of incorporating a more "realistic" model in the spirit of RICE (Nordhaus and Yang 1996), CWS (Eyckmans and Bertrand 2000), 
WITCH (Bosetti et al. 2007, 2008), or others that account for the nonlinearity of carbon emissions due to endogenous technological change, investment in renewable energies and other factors.

\subsection{Catastrophic Climate Change}

Greenhouse gases accumulated in the atmosphere disturb the climate system. As a result, a proportion of the home output is forgone to neutralize the local impacts of global warming $\Delta T_{h}$. This gives the home welfare,

$$
u_{h}=\ln \frac{Q_{h}}{1+\psi \cdot \Delta T_{h}^{2}} .
$$

Our functional form of (4) is similar to Nordhaus and Yang (1996). Here $\psi$ is the climate damage parameter with positive value. $\Delta T_{h}$ is a logarithmic function of global carbon emissions,

$$
\Delta T_{h}=\alpha \cdot \beta \cdot\left[\ln \left(\frac{E_{h}+E_{f}}{\bar{E}}\right)+x_{h}\right] .
$$

In the first term of Eq. (5), $\alpha$ is the sensitivity of radiative forcing with respect to carbon emissions, ${ }^{6} \beta$ is the sensitivity of global mean temperature with respect to radiative forcing (see e.g., Myhre et al. 1998), and $\bar{E}$ is the target global emission level for climate stability. The logarithmic relationship implies that increased emissions have a progressively smaller warming effect. In the second term, $x_{h}$ is the uncertainty surrounding the local impacts of climate change. Similar to $w_{h}$, the realization of $x_{h}$ can be arbitrarily small or large, and it is unknown to the home policymaker.

Substituting Eqs. (1), (3) and (5), and using the assumption that the home and foreign countries are symmetric, we can reparameterize and approximate Eq. (4) for home welfare in the linear-quadratic form:

$$
u_{h} \approx r_{f}-\left(1+w_{h}\right) r_{h}-\frac{\phi}{2}\left(r_{f}-(1+\tau) r_{h}-\bar{e}+x_{h}\right)^{2},
$$

where $\frac{\phi}{2}=\psi \cdot \alpha \cdot \beta$, and $\bar{e}=\ln \frac{\bar{E}}{2}$. By Eq. (3) and its foreign counterpart, we have assumed that home and foreign emissions are 1 each under the business-as-usual (BAU) scenario without carbon taxes. Therefore, $\bar{e}$ is the logarithmic of the target-to-BAU ratio of global emissions. Symmetrically, the change of foreign welfare is as follows

$$
u_{f} \approx r_{h}-\left(1+w_{f}\right) r_{f}-\frac{\phi}{2}\left(r_{h}-(1+\tau) r_{f}-\bar{e}+x_{f}\right)^{2} .
$$

As is clear from Eq. (6) and its foreign counterpart (7), when choosing carbon taxes the policymakers must trade off the climate damages avoided and the abatement costs incurred, while at the same time being concerned about the robustness of their policies.

\footnotetext{
6 For simplicity, here we have assumed a linear relationship between carbon emissions and atmospheric concentration.
} 
There are likely to be two types of carbon leakages. When the foreign carbon tax is low, the home policymaker will find it difficult to deliver ambitious emission reduction without losing export advantage to the foreign country. When the foreign carbon tax is high, the home policymakert will again find it hard to do so without being exposed to amplified market shocks. Collectivism could be potentially profitable, but this is not necessarily the case.

\subsection{Strategic Interactions with Concerns about Robustness}

We assume that home and foreign policymakers are faced with a two-stage game of international climate policy. In the first stage, the policymakers choose their negotiating positions, namely, unilateralism or collectivism. In the second stage, if collectivism has been chosen, they jointly maximize global welfare to obtain

$$
\max _{r_{h}, r_{f}}\left\{u_{h}+u_{f}\right\}
$$

Otherwise, they maximizes national welfare independently to obtain

$$
\max _{r_{h}}\left\{u_{h}\right\}+\max _{r_{h}}\left\{u_{f}\right\}
$$

To mimic the institution of a legally-binding international climate agreement, we rule out the possibility of renegotiation in the second stage of the game. An example for this setting is the Kyoto Protocol. ${ }^{7}$ The Protocol was adopted on 11 December 1997 and entered into force on 16 February 2005. It sets binding emission reduction targets for 37 industrialized countries in its first commitment period 2008-2012. The time lag between decision-making, action, and impacts realization puts international climate policies under large uncertainties.

With the consequences of their policy choices not being clear, policymakers fear that the worst-case scenario, if realized, may be dire. To model such beliefs, we follow the theory of minimax robust optimal control to assume the existence of a fictitious player called nature (see e.g., Zhou et al. 1996; Basar and Bernhard 2008; Basar and Olsder 1999; Hansen and Sargent 2008; Funke and Paetz 2011). Given the home and foreign taxes ( $r_{h}$ and $r_{f}$ ), nature strategically chooses the least-favorable combination of climatic and economic events $\left(w_{h}, w_{f}, x_{h}\right.$ and $\left.x_{f}\right)$ to minimize home and foreign welfare due to carbon pricing, $\min _{w_{h}, x_{h}}\left\{u_{h}\right\}$ and $\min _{w_{f}, x_{f}}\left\{u_{f}\right\}$ or equivalently (because $u_{h}$ is independent of $w_{f}, x_{f}$; and vice versa),

$$
\min _{w_{h}, w_{f}, x_{h}, x_{f}}\left\{u_{h}+u_{f}\right\}
$$

In the case that policymakers increase the taxes $\left(r_{h}\right.$ and $\left.r_{f}\right)$, nature will decrease the impacts of climate change $\left(x_{h}\right.$ and $\left.x_{f}\right)$ but increase the costs of mitigation $\left(w_{h}\right.$

\footnotetext{
7 See http://unfccc.int/essential_background/kyoto_protocol/items/1678.php.
} 
and $w_{f}$ ); and vice versa. This captures the policymakers' concerns about worst-case climate and economic outcomes.

It must be noted that in Eq. (10), $u_{h}+u_{f}$ is unbounded from below on the domains of $w_{h}, w_{f}, x_{h}$ and $x_{f}$, implying unlimited uncertainties and no policy solutions to Eqs. (8) or (9). This is unrealistic. Although not having a probability distribution of uncertain events, the policymakers still has confidence in the maximum impact of a policy. Therefore, a "soft-constrain" needs to be added to Eq. (10), forming

$$
\min _{w_{h}, w_{f}, x_{h}, x_{f}}\left\{u_{h}+u_{f}-\frac{\lambda_{1}}{2} w_{h}^{2}-\frac{\lambda_{1}}{2} w_{f}^{2}-\frac{\lambda_{2}}{2} x_{h}^{2}-\frac{\lambda_{2}}{2} x_{h}^{2}\right\} .
$$

Here, $\lambda_{1}>0$ and $\lambda_{2}>\phi$, which ensures that $y_{h}+y_{f}$ is strictly convex on the domains of $w_{h}, w_{f}, x_{h}$ and $x_{f}$, and therefore the minimum exists.

To understand the realistic meanings of the parameters $\lambda_{1}$ and $\lambda_{2}$, we solve Eq. (11) to derive the folowing worst-case shocks (given home and foreign taxes, $r_{h}$ and $r_{f}$ ),

$$
\begin{aligned}
x_{h} & =a \cdot\left(r_{f}-(1+\tau) \cdot r_{h}-\bar{e}\right), \\
x_{f} & =a \cdot\left(r_{h}-(1+\tau) \cdot r_{f}-\bar{e}\right), \\
w_{h} & =b \cdot r_{h}, \\
w_{f} & =b \cdot r_{f} .
\end{aligned}
$$

Here $a=\phi /\left(\lambda_{2}-\phi\right), b=1 / \lambda_{1}$, and there exists a one-to-one relationships between the parameterization of $\lambda_{1}$ and $\lambda_{2}$ and the values of $a$ and $b$. These shocks will be factored into the policymakers' choice of carbon taxes as per Eqs. (8) and (9). In other words, they are the worst-case shocks that policymakers have controlled for.

We see that the scales of $\lambda_{1}$ and $\lambda_{2}$ are negatively related to those of $a$ and $b$, which are in turn proportional to the sizes of $w_{h}, w_{f}, x_{h}$ and $x_{f}$. In the extreme case when $\lambda_{1}$ and $\lambda_{2}$ are infinite, we have $a=b=0$, and $w_{h}=w_{f}=x_{h}=x_{f}=0$. This implies that policymakers are sure of the consequences of their carbon policies. As $\lambda_{1}$ and $\lambda_{2}$ decrease, $a$ and $b$ will increase, and so will $w_{h}, w_{f}, x_{h}$ and $x_{f}$. This implies that policymakers have less confidence in the impacts of their carbon policies. They anticipate more adverse outcomes, and will therefore choose policies that are robust under the worst-case scenario.

It turns out from above that $\lambda_{1}$ and $\lambda_{2}$ are negatively correlated with the policymakers' concerns about worst-case climate and economic outcomes, as measured by $a$ and $b$. We shall investigate the policy implications for various $a$ and $b$ values (or equivalently, various parameterizations of $\lambda_{1}$ and $\lambda_{2}$ ) shortly.

\subsection{Policy Equilibria}

Given the socio-economic system described above, a three-player two-stage game of international climate policy can be formulated, which includes the home and foreign policymakers, and the fictitious player nature. We consider the (pure strategy) subgame perfect equilibrium such that 
Table 1 Types of equilibria in the game of international climate policy

\begin{tabular}{lll}
\hline & Collectivism & Unilateralism \\
\hline Higher carbon taxes & Type 1 & Type 4 \\
Lower carbon taxes & Type 3 & Type 2 \\
\hline
\end{tabular}

- given the least-favorable market and climate shocks, the policymakers' negotiating positions and carbon taxes maximize the home and foreign welfare, respectively;

- given the policymakers' negotiating positions and the least-favorable market and climate shocks, the carbon taxes maximize the home and foreign welfare, respectively.

Depending on the policymaker's negotiation position and carbon taxes, we anticipate four types of equilibria to arise from the game. In Type 1 equilibrium, policymakers act collectively and impose higher carbon taxes than they do unilaterally; in Type 2 equilibrium, policymakers act unilaterally and impose lower carbon taxes than they do collectively; in Type 3 equilibrium, policymakers act collectively but impose lower carbon taxes than they do unilaterally; in Type 4 equilibrium, policymakers act unilaterally but impose higher carbon taxes than they do collectively. The four types of equilibria are summarized in Table 1.

It is clear that the equilibrium could be solved by backward induction. The existence and uniqueness of equilibria in the second stage sub-game follows from Moré (1974) and Facchinei et al. (2007). Subsequently, by comparing welfare levels of unilateralism and collectivism, the policymakers' choice of negotiating positions at the first stage sub-game can be calculated.

In the following section, we explore the relationship between the policymakers' concerns about worst-case climate and economic outcomes and their policy choices.

\section{Numerical Simulation and Discussion}

In this section we report the numerical results of a calibrated game of international climate policy. In this exercise, the price elasticity of energy demand, $\tau$ in Eq. (3), is set equal to 0.5 . It implies that a $1 \%$ increase of price will result in a $0.5 \%$ reduction of energy consumption and carbon emissions. This is consistent with most existing estimates in the literature (see e.g., Stern 2012). The parameter $\phi$ in Eqs. (6) and (7) is set equal to 0.4. It implies that a doubling of global emissions from the target level increases global temperature by $2^{\circ}-4^{\circ}$, which leads to an annual GDP loss of no more than $10 \%$. This is consistent with the estimations of Stern (2007) and Nordhaus (2008). The target-to-BAU-emissions ratio in Eqs. (6) and (7) is set equal to $16 \%$ (i.e., $\bar{e}=-1.81)$. This ratio is calibrated to mimic the shift of global carbon concentration pathway from a BAU scenario (Riahi et al. 2011) to an aggressive mitigation scenario that keeps global mean temperature increase below $2^{\circ}$ (Vuuren et al. 2011) as adopted by the Intergovernmental Panel on Climate Change for its fifth assessment report. 
Carbon Tax

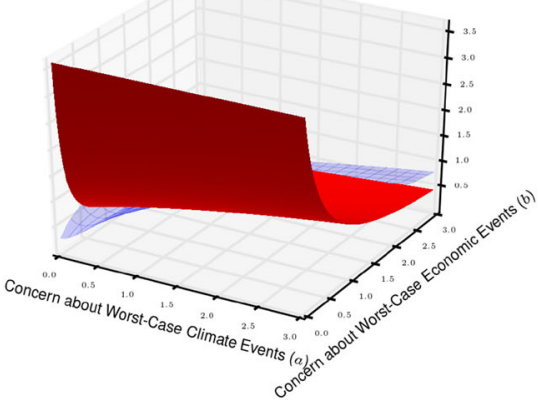

Worst-Case Economic Shocks Controlled $(w)$

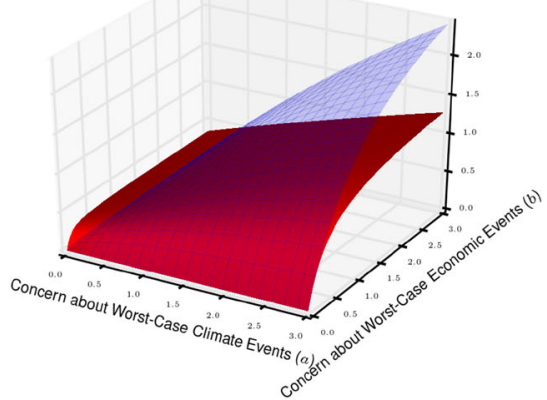

Worst-Case Climate Shocks Controlled $(x)$

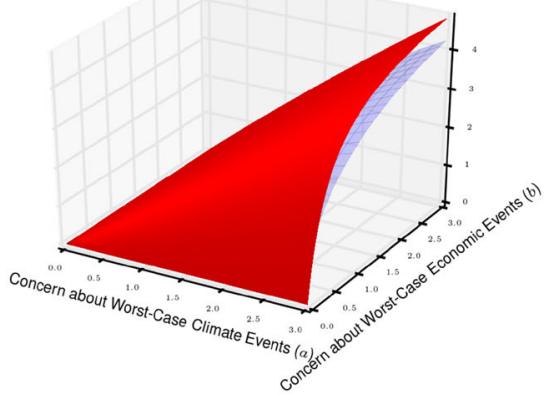

Welfare Gain from Collectivism

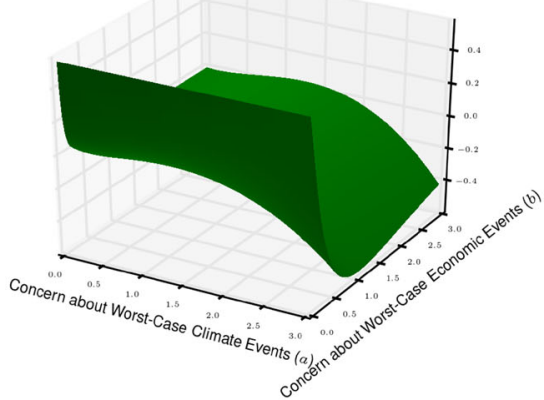

Fig. 1 Comparison between collectivism and unilateralism. Note in the upper and lower-left panels, the red surface denotes "Collectivism" and the blue surface with strips denotes "Unilateralism" (colour figure online)

We have simulated the policymakers' choice of carbon taxes as per Eqs. (8) and (9), and the worst-case climate and economic events that they have controlled for as per Eqs. (12) to (15) under the collective and the unilateral international policy regimes, respectively, for various combinations of $a$ and $b$ values. We have also calculated the potential welfare gain from collectivism as per Eqs. (6) and (7). These results are reported in the four panels of Fig. 1. Because the home and foreign countries are symmetric, only one set of results is reported without being labelled as home or foreign. Overall, our results contridict the model predictions of Ulph and Ulph (1997) and Funke and Paetz (2011) that uncertainty justifies strong mitigation action and facilitates international policy coordination.

In Fig. 1, the upper-left panel shows that policymakers are more likely to take up aggressive carbon policies under a collective international regime when they are more concerned about worst-case climate events (catastrophe) than worst-case economic events (mitigation costs). But this is not the case as policymakers become increasingly more concerned about the economic costs of mitigation. When the concerns about worst-case climate and economic events are both large, policymakers will only impose high carbon taxes unilaterally. Underpining carbon taxes are the policymakers' control for unfavorable climate and economic outcomes. As the upper-right panel shows, higher carbon taxes allow policymakers to keep climate uncertainty in check 


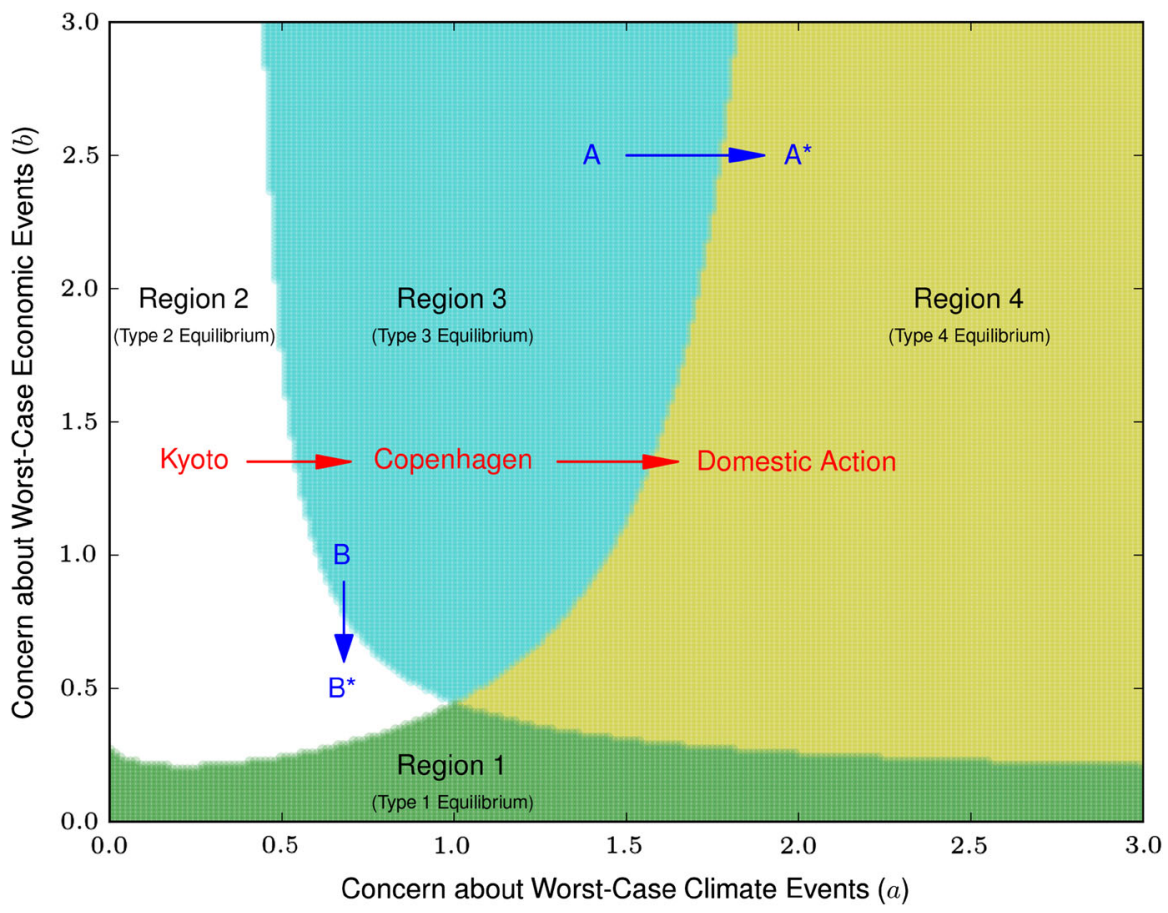

Fig. 2 Equilibrium with various policy concerns. Note Region 1 quantifies the $a$ and $b$ values such that collectivism is welfare-improving and leading to higher carbon taxes; Region 2 quantifies the $a$ and $b$ values such that collectivism is welfare-improving but leading to lower carbon taxes; Region 3 quantifies the $a$ and $b$ values such that unilateralism is welfare-improving and leading to lower carbon taxes; Region 4 quantifies the $a$ and $b$ values such that unilateralism is welfare-improving but leading to higher carbon taxes

and reduce the severity of climate catastrophe under the least favorable scenario. However, as the lower-left panel shows, higher carbon taxes also expose policymakers to larger economic uncertainty and increase the magnitude of output loss under the least favorable scenario. In general, we don't see from above a clear connection between the policymaker's negotiation position, carbon taxes, and the control for climate and economic uncertainties. As a result, the welfare implications of collectivism are quite mixed as the lower-right panel shows.

To further understand the making of international climate policy under uncertainties, we have simulated equilibria of the game as defined in the last section for various $a$ and $b$ values. The results are reported in Fig. 2, where we divide the quadrant of $a$ and $b$ values into four regions, each supporting a type of equilibrium.

In Region 1, policymakers are more concerned about the worst-case climate outcome. They anticipate that the consequences of climate change under the worst case scenario is dire, and believe that the payoff of mitigation is clearly positive. Collective action allows both countries to impose higher carbon taxes and to constrain the impacts of least-favorable climate shocks. So policymakers will consider collectivism as a way to improve national welfare. This corresponds to the Type 1 equilibrium listed in Table 1, and it is consistent with the finding of Barrett and Dannenberg (2012) that 
the incentive to coordinate is strong where threshold uncertainty surrounding the payoff of mitigation is small.

In Region 2, policymakers are more concerned about the worst-case economic outcome. They worry that carbon taxes could damage their competitive advantage in the global trade market and reduce their national welfare. So policymakers prefer to impose lower carbon taxes and to stay away from any international climate agreements. This corresponds to the Type 2 equilibrium, and it is consistent with the recent finding of Barrett (2013) that international negotiation will fail if the probability of climate catastrophe is underestimated by individual players.

In Region 3, policymakers are in a dilemma. To some extent they are aware of the catastrophic consequences of climate change, but what worries them more is the economic uncertainty surrounding the costs of carbon mitigation. They are willing to enter into an international agreement but are just not ready to commit more mitigation efforts than they would do unilaterally. This corresponds to the Type 3 equilibrium. Barrett and Dannenberg (2014) have provided experimental evidence that the fear of crossing a dangerous threshold can favor coordination in climate negotiations, but uncertainty about the location of the threshold will push the players back to a prisoners' dilemma. This region potentially explains the paradox of why countries would agree to a collective goal, aimed at reducing the risk of catastrophe, but act as if they were blind to this risk.

In Region 4, policymakers are most stressed. They have large concerns about the catastrophic consequences of climate change as well as the economic costs of carbon mitigation. They anticipate that the hardship of collective policy will undermine their mitigation efforts, and therefore prefer to act along and impose higher carbon taxes before an international binding agreement is reached. This corresponds to the Type 4 equilibrium, and it appears to support the view of Vasconcelos et al. (2013) that "a polycentric approach involving multiple institutions is more effective than that associated with a single, global one".

\section{Policy Implications}

By construction, the global welfare as calculated in the first stage of the game (simply, national welfare by two) is maximized in each of the equilibria as represented by the four regions in Fig. 2, whether policymakers are precommited to coordinate carbon taxes in the second stage or not. So there is actually no failure of policy negotiation in terms of welfare. In other words, forcing governments into a binding agreement could worsen global welfare due to the contagion of unfavorable economic shocks. In particular, when governments are situated in Region 4, collectivism could even lead to insufficient carbon reductions. Together, the results suggest the inappropriateness of measuring the success of an international climate agreement by its engagement of countries.

The existence of Type 3 equilibrium (Region 3) implies that there is no correlation between the policymakers' concern about robustness and the desirability of collectivism. To see this, suppose that governments are originally situated at point $A$. The increase of concern about worst-case climate events (moving from $A$ to $A^{*}$ ) breaks down the existing policy alliance, but it results in more aggressive mitigation. Alter- 
natively, suppose that governments are originally at point $B$. The increas of concern about worst-case economic events (moving from $B$ to $B^{*}$ ) renders collectivism undesirable until finally a Type 1 equilibrium (Region 1 ) is reached. Indeed, many more results can be obtained by the same line of reasoning.

The numerical results above seem to provide a possible explanation to political dynamics at the UNFCCC Conferences of Parties. In the 1990's when the risk of climate change was under-estimated, the fear of economic uncertainty prevailed (originally in Region 2) and as a consequence the Kyoto Protocol was not able to engage all major fossil-fuel-burning countries at that time and to thereby cover a sufficient proportion of the global carbon emissions. Nowadays the worry of catastrophic climate change grows. However, because little policy experimentation has been carried out over in the last decade to test the responses of the global economy to carbon pricing of any form, the concern about economic costs of mitigation stay unresolved (moving into Region 3). Governments thus favor collectivism but end up with minor mitigation efforts as they would do unilaterally.

\section{Concluding Remarks}

This paper argues that the theoretical framework which is based on maximizing expected utility is problematic in the analysis of international climate policies when the issues associated with climate change are historically unprecedented, and when policymakers do not have a prior distribution over possible outcomes. It proposes a theoretical model that extends the "maxmin" approach to address policymakers' concern about policy robustness in the least-favorable climate and economic scenarios the making of international climate policies. Under the alternative assumption that policymakers act strategically but choose the policy that incurs the highest possible gain in the worst-case scenario, collectivism is shown to be not generally optimal. Indeed, the paper provides numerical examples where unilateralism is superior for both carbon mitigation and economic loss minimization. Hence, it is not appropriate to judge the success of global climate talks by the extent of country engagement or each country's reduction commitments.

The model presented here not only improves our understanding of the current deadlock in international climate change negotiations, it also allows us to highlight ways in which the development of global carbon mitigation agendas could move forward. One important implication of this study is that the approach of climate policy negotiation should be both gradual and experimental with different policy interventions to enable learning and should focus on mechanisms that reduce economic uncertainty. One such device might be a "safety valve" that could be made available in order to truncate the negative impact of unexpected market shocks (McKibbin and Wilcoxen 2002). Under the McKibbin-Wilcoxen blueprint, for instance, a hybrid policy that combines a fixed number of tradable, long-term emissions permits with an elastic supply of short-term permits would ensure that the abatement done within a given country would be done at minimum cost. In addition, the focus of talks on climate change should be on the resolution of uncertainties, especially those related to the economic cost of mitigation policies. In this respect, what have been achieved in 
the recent UNFCCC Conferences of the Parties should be considered as successful. Although still limited, the concrete actions that have been promised by the participating countries allow experimentation and thus the generation of knowledge of the nature of this policy problem. This should reduce uncertainty around the economic costs of policies and facilitate more cooperative and ambitious mitigations in the future.

Acknowledgments The authors gratefully acknowledge support from Australian Research Council Discovery Grant DP0988281.

\section{References}

Basar T, Bernhard P (2008) $\mathrm{H}^{\infty}$ optimal control and related minimax design problems: a dynamic game approach, 2nd edn. Birkhäuser, Boston

Basar T, Olsder GJ (1999) Dynamic noncooperative game theory, 2nd edn. SIAM, Philadelphia, pp 342-350

Barrett S (2013) Climate treaties and approaching catastrophes. J Environ Econ Manag 66:235-250

Barrett S, Dannenberg A (2012) Climate negotiations under scientific uncertainty. Proc Natl Acad Sci USA 109(43):17372-17376

Barrett S, Dannenberg A (2014) Sensitivity of collective action to uncertainty about climate tipping points. Nat Clim Chang 4:36-39

Bosetti V, Massetti E, Tavoni M (2007) The WITCH model: structure, baseline, solutions. Fondazione Eni Enrico Mattei. NOTA DI LAVORO 10.2007

Bosetti V, Tavoni M, De Cian E, Sgobbi A (2008). The 2008 WITCH model: new model features and baseline. Fondazione Eni Enrico Mattei. NOTA DI LAVORO 85.2009

Brainard WC (1967) Uncertainty and the effectiveness of policy. Am Econ Rev 57(2):411-425

Dornbusch R (1976) Expectations and exchange rate dynamics. J Polit Econ 84(6):1161-1176

Eyckmans J, Bertrand, C (2000) Integrated assessment of carbon and sulphur emissions, simulations with the CLIMNEG model. CLIMNEG Working Paper No. 32 and ETE Working Paper No. 8

Facchinei F, Fischer A, Piccialli V (2007) On generalized nash games and variational inequalities. Oper Res Lett 35(2):159-164

Field CB, Barros VR, Mastrandrea MD et al (2011) Climate change 2014: impacts, adaptation, and vulnerability: summary for policymakers. The Intergovernmental Panel on Climate Change (IPCC)

Froyn CB (2005) Decision criteria, scientific uncertainty, and the global warming controversy. Mitig Adapt Strateg Glob Chang 10:183-211

Funke M, Paetz M (2011) Environmental policy under model uncertainty: a robust optimal control approach. Clim Chang 107:225-239

Hall JM, Lempert R, Keller K, Hackbarth A, Mijere C, McInerney D (2012) Robust climate policies under uncertainty: a comparison of Info-Gap and RDM methods. Risk Anal 32(10). doi:10.1111/j. 1539-6924.2012.01802.x

Hansen LP, Sargent TJ (2008) Robustness. Princeton University Press, New Jersey, pp 25-52

Hoel M (1991) Global environmental problems: the effects of unilateral actions taken by one country. J Environ Econ Manag 20(1):55-70

Jiménez-Lizárraga M, Poznyak A (2007) Robust Nash equilibrium in multi-model LQ differential games: analysis and extraproximal numerical procedure. Optim Control Appl Methods 28:117-141

Jiménez-Lizárraga M, Poznyak A (2012) Necessary conditions for robust Stackelberg equilibrium in a multi-model differential game. Optim Control Appl Methods 33:595-613

Kunreuther H, Heal G, Allen M, Edenhofer O, Field CB, Yohe G (2013) Risk management and climate change. Nat Clim Chang 3:447-450

McInerney D, Lempert R, Keller K (2012) What are robust strategies in the face of uncertain climate threshold responses? Clim Chang 112:547-568

McKibbin WJ, Wilcoxen PJ (2002) The role of economics in climate change policy. J Econ Perspect 16(2):107-129

McKibbin W, Wilcoxen P (2013) A global approach to energy and the environment: the G-cubed model. Handbook of CGE modeling, vol 17. North Holland, USA, pp 995-1068

Moré JJ (1974) Coercivity conditions in nonlinear complementarity problems. SIAM Rev 16(1):1-16 
Myhre G, Highwood EJ, Shine KP, Stordal F (1998) New estimates of radiative forcing due to well mixed greenhouse gases. Geophys Res Lett 25(14):2715-2718

Nordhaus WD (2008) A question of balance. Yale University Press, New Haven

Nordhaus WD, Yang Z (1996) A regional dynamic general-equilibrium model of alternative climate-change strategies. Am Econ Rev 86(4):741-765

Riahi K, Rao S, Krey V, Cho C, Chirkov V, Fischer G, Kindermann G, Nakicenovic N, Rafai P (2011) RCP 8.5-a scenario of comparatively high greenhouse gas emissions. Clim Chang 109:33-57

Stern D (2012) Interfuel substitution: a meta-analysis. J Econ Surv 26:307-331

Stern N (2007) The economics of climate change: the Stern review. Cambridge University Press, Cambridge

Uzawa H (2003) Economic theory and global warming. Cambridge University Press, Cambridge

Ulph A, Ulph D (1997) Global warming, irreversibility and learning. Econ J 107(442):636-650

van den Broek W, Engwerda J, Schumachar J (2003) Robust equilibria in indefinite linear-quadratic differential games. J Optim Theory Appl 119(3):565-595

Vasconcelos VV, Santos FC, Pacheco JM (2013) A bottom-up institutional approach to cooperative governance of risky commons. Nat Clim Chang 3:797-801

Vuuren D, Stehfest E, den Elzen M, Kram T, van Vliet J, Deetman S, Isaac M, Goldewijk K, Hof A, Beltran A, Oostenrijk R, van Ruijven B (2011) RCP2.6: exploring the possibility to keep global mean temperature increase below $2{ }^{\circ} \mathrm{C}$. Clim Chang 109:95-116

Weitzman ML (2012) GHG targets as insurance against catastrophic climate damages. J Public Econ Theory 14(2):221-244

Zhou K, Doyle JC, Glover K (1996) Robust and optimal control. Prentice Hall, New Jersey, pp $413-447$ 